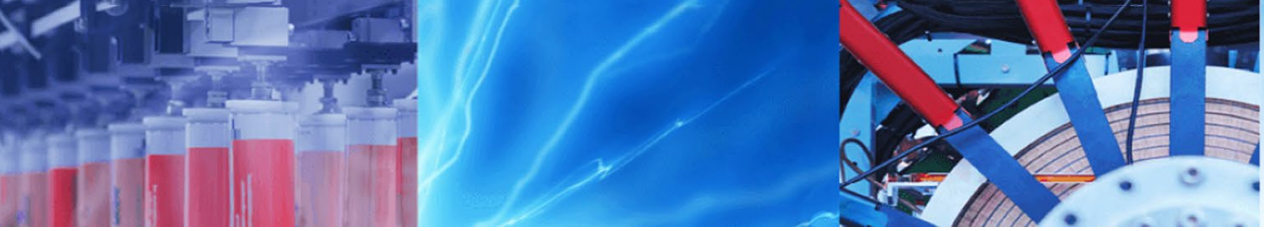

Research Article

\title{
CS-means: Determining optimal number of clusters based on a level-of-similarity
}

\author{
Rabindra Lamsal ${ }^{1}$ (1) $\cdot$ Shubham Katiyar ${ }^{1}$
}

Received: 28 May 2020 / Accepted: 22 September 2020 / Published online: 6 October 2020

(c) Springer Nature Switzerland AG 2020

\begin{abstract}
This paper proposes a centroid-based clustering algorithm, cs-means, which is capable of clustering data-points with $\mathrm{n}$-features, without having to specify the number of clusters to be formed. The core logic behind the algorithm is a similarity measure that collectively decides whether to assign an incoming data-point to a pre-existing cluster, or create a new cluster and assign the data-point to it. The algorithm is application-specific and applicable when the need is to perform clustering analysis of a stream of data-points, where the similarity measure between an incoming data-point and the cluster to which the data-point is to be associated with, is higher than the predefined level-of-similarity (cluster strictness). The algorithm was experimented on 4 public datasets and 10 isotropic Gaussian blobs. The cluster analysis strongly confirms the objectives of the proposed clustering algorithm.
\end{abstract}

Keywords Unsupervised $\cdot$ Clustering $\cdot$ Centroid-based.

\section{Introduction}

The main gist behind clustering is to group data-points into various groups (clusters) based on their features, i.e. properties. The generation of clusters varies applicationwise [17], because it depends on what factors are to be taken into consideration to form a particular cluster. But, the focus of every clustering algorithm remains same, i.e. to group similar data-points to a common cluster. The thing that differs is how this goal of forming a cluster is achieved. Different algorithms use different concepts to deal with similarity measure among the data-points. There are many popular clustering algorithms that group datapoints based on various strategies to define the similarity measure between them. Centroid-based [9], densitybased [7], graph-based [10], etc. are the commonly used strategies.

Often used algorithms like k-means, hierarchical clustering, DBSCAN, etc. require a set of data-points in space, beforehand. Without making some adjustments to these pre-existing clustering algorithms, it is not possible to cluster a stream of real-time data-points. A real-world problem exists when there is a necessity of grouping a set of datapoints but the possible number of clusters the data-points can generate is unknown. Therefore, considering the realtime approach limitation of the algorithms mentioned above, this paper proposes a clustering algorithm that can group incoming data-points without having to initialize the number of clusters to be formed. Hereinafter, the proposed clustering algorithm is termed as CS-means, with "cs" standing for "cluster strictness". The main objectives of the proposed algorithm are to: (1) facilitate those problems which require clustering of data-points based on some predefined level-of-similarity, (2) introduce a realtime approach to a centroid based clustering algorithm, (3) determine the optimal number of clusters for a dataset. In the literature review, we did not come across any literary works that had exploited the level-of-similarity

Rabindra Lamsal, rabindralamsal@outlook.com; Shubham Katiyar, shubha25_scs@jnu.ac.in | ${ }^{1}$ School of Computer and Systems Sciences, Jawaharlal Nehru University, New Delhi 110067, India. 
concept for generating clusters. However, there have been few approaches to generation of clusters of data streams. D-Stream [5] used a density-based approach to generate and adjust clusters in realtime. [1] divided the whole clustering process into two components: online and offline. Online component periodically stored detailed summary statistics and offline component facilitated an analyst to use variety of inputs including time horizon and number of clusters to be formed. Work in [8] maintained clustering of a sequence of data-points observed at a period of time. In [3], based on the work of [2], the authors proposed an algorithm for real-time data analytics that works based on the eccentricity of each sample to all existing data and existing clusters. Similarly, some notable works have been done in the area of determining optimal numbers of clusters. In [15], the authors use U-Control Chart (UCC) method with Automatic Clustering using Differential Evolution $(A C D E)[6,11]$ to determine the number of clusters in k-means. [13] presented the Spectral Global Silhouette method, to calculate the optimal number of clusters in a dataset using a Spectral Clustering algorithm. In [16] outliers were detected and removed to better estimate the optimal number of clusters when there are more variables than objects. In [12], a cluster validity evaluation method based on the ratio of deviation of sum-of-squares and Euclidean distance was proposed, which determines the near-optimal number of clusters.

The paper is organized as follows. Section 2 describes the core logic of the proposed algorithm. Implementation of the algorithm is done in Sect. 3. Section 4 discusses the experimental results and Sect. 5 concludes the paper.

\section{The $C S$-means algorithm}

The cs-means algorithm requires an initial declaration of cluster strictness. Cluster strictness is the lowest permitted level-of-similarity between a data-point and the centroid of a cluster. Here, cluster centroid is the average of features of the data-points present in a cluster and is an effective way of representing a particular cluster. If cluster strictness is set to a measure of 60 , then the accepted variability between a cluster centroid (C) and a data-point (D) should be a measure of 40 at-most, for $D$ to be associated to cluster C.

Hence, the cs-means algorithm is capable of generating clusters based on a level-of-similarity. If one needs clusters with very low variance among the data-points, the cluster strictness can be adjusted accordingly; maybe around $80-95 \%$. That is why cluster strictness plays a significant role in generating clusters, and its value depends entirely on what application the clustering is being done.

Algorithm 1, is the pseudo-code for the proposed CSmeans clustering algorithm.

\subsection{Data-point and features}

A data-point can have multiple features ( $\mathrm{n}$-features). Those features are the characteristics which collectively define the data-point. A stream of data-points can be grouped into various clusters, based on their features. Below is an example of a data-point with 7 features.

A data-point : 5101520253035

During the implementation of the cs-means algorithm, the above pattern is used to represent a data-point, and similarity measure between the data-points and cluster(s) is calculated accordingly. The algorithm takes data-points with $\mathrm{n}$-features, one data-point at a time, and requires all the data-points to have the same fixed number of features. When the algorithm runs for the very first time and is waiting for a new data-point; the number of cluster(s) is 0 . Cluster strictness needs to be defined beforehand. Based on the requirements, this value can be adjusted. Higher the value of cluster strictness, less is the variance between the data-points in a cluster. Depending on the data-points, using a higher value of cluster strictness result in a large number of clusters. This is more likely to happen if the incoming data-points are less identical to each other.

After assigning some value to cluster strictness, say $70 \%$, the number of features that should be matched between a data-point and a cluster is calculated using Formula 1.

should_match_features $=\frac{\text { no_of_features } * \text { cluster_strictness }}{100}$

Suppose, the data-points that are to be clustered have 20 features. That means cluster strictness with $70 \%$ results into 14 features that must be matched (at least) for a datapoint to be associated with a particular cluster.

\subsection{Qualifying feature}

A feature qualifies to be counted as a matched feature when its similarity measure lies between the range: (cluster_strictness) and (100 + (100 - cluster_strictness $))$. That is, the valid range is $70-130$ when cluster strictness is considered 70 . Basic mathematics can be used when variability check of 5 and 13 is to be done with respect to 9.9 with respect to 9 gives a similarity measure of 100.5 with respect to 9 gives a similarity measure of 55.56. And, 13 with respect to 9 gives a similarity measure of 144.44 . If allowed variability is considered to be a measure of 50, then similarity measure of both 5 and 13 falls under the range $50-150$, and are considered to be associated to 9 by at-least a measure of 50 .

In the case of data-points, Formula 2 is used to calculate the similarity measure. 
$S(C i, F j))=\frac{100 * \text { datapoint }_{j}}{\text { centroid }(i, j))}$

where $i$ is an index for clusters, and $j$ is an index for features.

As an example, in Formula 2, $i=3$ and $j=7$ reflect the fact that cluster 3 is being considered, and 7 th feature of a datapoint and 7th feature of the centroid of cluster 3 are being used to compute the level-of-similarity between them. If the resulted similarity measure lies between the range (cluster_strictness) and $(100+(100$ - cluster_strictness $))$, then the 7 th feature is said to be matched, and the value of matched feature counter for the cluster 3 is incremented by 1 . This process of calculating the similarity feature of an incoming data-point is done with all the cluster(s). And if the matched feature counter for a cluster reaches the limit of the minimum number of features that should be matched between a data-point and a cluster, that particular cluster is now placed into a list of qualified clusters.

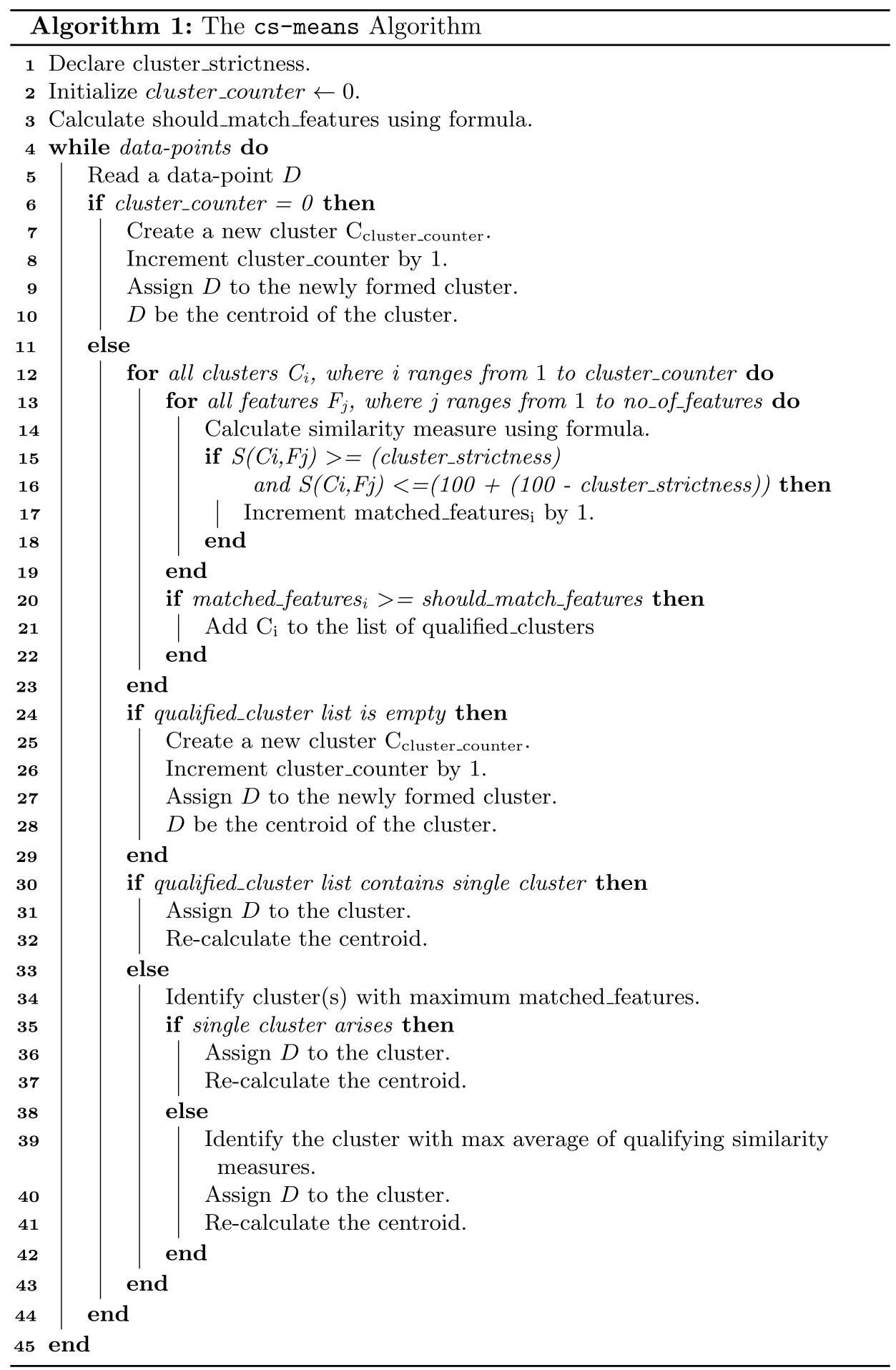




\subsection{Qualified cluster list}

This list contains the cluster(s) that satisfy the condition of having at least a minimum number of features that have matched with an incoming data-point. After calculation of similarity measure for a data-point with all the cluster(s), there might arise any one of the following three situations.

\subsubsection{A qualified cluster list is empty}

The qualified list being empty indicates that no similar cluster(s) were found within the provided level-of-similarity. Hence, a new cluster is generated, and the data-point is assigned to the newly formed cluster. The data-point is the centroid of this cluster.

\subsubsection{A qualified cluster list contains exactly 1 cluster}

In this case, the data-point is simply assigned to the cluster which is present in the list. And, the centroid of the cluster is re-calculated.

\subsubsection{A qualified cluster list contains more than 1 cluster}

Case 1: If the list contains more than 1 cluster, the cluster with maximum matched features is identified. The data-point is now assigned to the identified cluster. Case 2: Sometimes, two or more cluster might come-up with the same highest number of matched features. In this case, the cluster with a maximum average of qualifying similarity measures is identified, and the data-point is assigned to that cluster accordingly.

\subsection{Conflicting clusters}

Whenever multiple clusters show up in qualified cluster list, while trying to identify the nearest similar cluster based on the highest number of matched features, those clusters are said to be the conflicting ones. In such case, an average of qualifying similarity measures is calculated for all the clusters which are in the qualified list and have the same number of matched features, and finally, the cluster with the maximum average is considered to be the cluster to which the data-point is associated. While calculating an average, only the qualifying similarity measures are being considered. One fact is also being taken into consideration that technically 60 and 140 are same with respect to 100 . Both have a variability measure of 40 .

\section{Implementation of the $\mathrm{CS}$-means algorithm}

Let us consider the following six data-points, each of 10 features, that are to be clustered. The data-points are input to the algorithm in a serial fashion.
Data-point 1 : 10152025303540455055

Data-point 2 : 09351845103260411020

Data-point 3 : 18131827303838414957

Data-point 4 : 20201805153450431050

Data-point 5 : 17171815223544431053

Data-point 6 : 10322045125540550925

Let us suppose, level-of-similarity for a cluster is considered to be 60 . That means variability measure of 40 between a data-point and a cluster centroid is acceptable. With cluster strictness set to 60 , using formula 1 we get minimum number features that must be matched to be 6 .

Initially, when Data-point 1 is input to the algorithm, because of the absence of cluster(s), a new cluster $\mathrm{C} 1$ is created, and Data-point 1 is assigned to it, and features of Data-point 1 is assumed to be the centroid of $\mathrm{C} 1$.

C1 = Data-point 1

C1 : 10152025303540455055

For Data-point 2

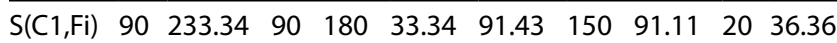

The number of qualified features is 4 , which is less than 6 . $\mathrm{C} 1$ cannot be added to the list of qualified clusters. Since there are no clusters in the list of qualified clusters, a new cluster $\mathrm{C} 2$ is generated, and Data-point 2 is assigned to $C 2$.

$\mathrm{C} 2$ = Data-point 2

C2 : 09351845103260411020

For Data-point 3

$\begin{array}{lllllllllll}\mathrm{S}(\mathrm{C} 1, \mathrm{Fi}) & 180 & 86.67 & 90 & 108 & 100 & 108.57 & 95 & 91.11 & 98 & 103.64\end{array}$

$\begin{array}{lllllllllll}\mathrm{S}(\mathrm{C} 2, \mathrm{Fi}) & 200 & 37.14 & 100 & 60 & 300 & 118.75 & 63.33 & 100 & 490 & 285\end{array}$

Only $\mathrm{C} 1$ qualified to be in in the list of qualified clusters, Data-point 3 is assigned to $\mathrm{C} 1$.

C1 = Data-point 1 , Data-point 3

New C1 : 141419263036.5394349 .556

For Data-point 4

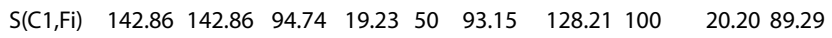

$\begin{array}{llllllllllll}\mathrm{S}(\mathrm{C} 2, \mathrm{Fi}) & 222.22 & 57.14 & 100 & 11.11 & 150 & 106.25 & 83.33 & 104.87 & 100 & 250\end{array}$

Since there no cluster qualified to be kept in the list of qualified clusters, a new cluster C3 is generated, and Datapoint 4 is assigned to $C 3$.

$\mathrm{C} 3$ = Data-point4

New C3 : 20201805153450431050 
For Data-point 5

\begin{tabular}{lllllllllll}
\hline $\mathrm{S}(\mathrm{C} 1, \mathrm{Fi})$ & 121.43 & 121.43 & 94.74 & 57.59 & 73.33 & 95.89 & 112.82 & 100 & 20.20 & 94.64 \\
$\mathrm{~S}(\mathrm{C} 2, \mathrm{Fi})$ & 188.89 & 48.57 & 100 & 33.33 & 220 & 109.38 & 73.33 & 104.88 & 100 & 265 \\
$\mathrm{~S}(\mathrm{C} 3, \mathrm{Fi})$ & 85 & 85 & 100 & 300 & 146.67 & 102.94 & 88 & 100 & 100 & 106 \\
\hline
\end{tabular}

Since $\mathrm{C} 1$ and $\mathrm{C} 3$ qualified to be in the list of qualified clusters and both the clusters have the same number of qualified features. Now, the average of the qualifying features is calculated for both the clusters. Qualifying features beyond 100 are scaled below 100 using Formula 3:

scaled value $=100-($ value beyond hundred -100$)$

Average of qualifying features $(\mathrm{C} 1)=87.87$

Average of qualifying features $(C 3)=93.63$

Since the average of qualifying features for $\mathrm{C} 3$ is higher, Data-point 5 is assigned to $C 3$.

C3 = Data-point 4, Data-point 5

New C3 : 18.518 .5181018 .534 .547431051 .5

For Data-point 6 scikit-learn library [14] was used to create those Gaussian blobs. The overview of the datasets used for experimentation is given in Table 1 and Table 2.

The 4 public datasets are generally associated with the classification task. The different classes within these datasets have been considered their respective centers in this experimentation for clustering task. No instances of these datasets have been removed except for the WPBC Dataset, which had a single unknown value in four of its samples. Therefore, the total number of samples in the WPBC Dataset was 194 after removing those four samples.

For cluster analysis, the intra-cluster distance was computed for all the datasets against the discrete values of the cluster strictness ranging from 5 to $95 \%$ with a $5 \%$ difference gap. Euclidean distance was used as the distance function for computing the total intra-cluster distance. For fair experimentation, each Gaussian blob has

\begin{tabular}{lllllllllll}
\hline $\mathrm{S}(\mathrm{C} 1, \mathrm{Fi})$ & 71.43 & 228.57 & 105.26 & 173.08 & 40 & 150.68 & 102.56 & 127.91 & 18.18 & 44.64 \\
$\mathrm{~S}(\mathrm{C} 2, \mathrm{Fi})$ & 111.11 & 91.43 & 111.11 & 100 & 120 & 171.88 & 66.67 & 134.15 & 90 & 125 \\
$\mathrm{~S}(\mathrm{C} 3, \mathrm{Fi})$ & 54.05 & 172.97 & 111.11 & 450 & 64.86 & 159.42 & 85.11 & 127.91 & 90 & 48.54 \\
\hline
\end{tabular}

Since C2 is the only cluster qualifying to be in the list of qualified clusters, Data-point 6 is assigned to C2.

C2 $=$ Data - point 2, Data - point 6

New C2 : 9.533 .519451143 .550489 .522 .5

The data-points were successfully grouped into 3 various clusters based on the similarity measure of their features. Cluster strictness of 60 was considered, hence permitted variability measure between a data-point and a cluster centroid was 40.

\section{Experimental results}

The cs-means algorithm was experimented on 14 different datasets for evaluation purposes. Among those datasets, 4 of them are popularly used public datasets [4], namely Iris Dataset, Wine Dataset, Wisconsin Diagnostic Breast Cancer (WDBC) Dataset, and Wisconsin Prognostic Breast Cancer (WPBC) Dataset. The remaining 10 datasets are the randomly generated isotropic Gaussian blobs of varying number of samples, features, and centers. The been generated with varying sizes, features, and centers. Figure 1 shows the results obtained from the 4 public datasets. Similarly, Fig. 2 shows the results obtained from the 10 isotropic Gaussian blobs. Each experimental result graph illustrates the effect of varying the value of cluster strictness on the formation of clusters of data-points. The blue plot represents the number of clusters formed against a value of cluster strictness. The red plot represents the total intra-cluster distance against a value of cluster strictness.

\subsection{Discussion}

The experimental results strongly confirm the main objectives of the cs-means algorithm. Figure 1 shows the results obtained from the real-world public datasets. As the cluster strictness is increased, the number of clusters also starts increasing. It is also evident that with the increase in the number of clusters, the intra-cluster distance improves. In those 4 public datasets, only a single cluster is formed until the value of cluster strictness reaches beyond a specific limit. In the Iris Dataset, there are three classes, and the clusters for these three classes 
Table 1 Overview of the 4 Public Datasets

\begin{tabular}{lllll}
\hline SN. & Dataset & \#samples & \#features & \#centers \\
\hline 1 & Iris Dataset & 150 & 4 & 3 (classes) \\
2 & Wine Dataset & 178 & 13 & 3 (classes) \\
3 & WDBC Dataset & 569 & 30 & 2 (classes) \\
4 & WPBC Dataset & 194 & 33 & 2 (classes) \\
\hline
\end{tabular}

Table 2 Overview of the 10 Isotropic Gaussian Blobs

\begin{tabular}{llccl}
\hline SN. & Dataset & \#samples & \#features & \#centers \\
\hline 1 & s1000_f100_c10 Dataset & 1000 & 100 & 10 \\
2 & s1000_f100_c50 Dataset & 1000 & 100 & 50 \\
3 & s5000_f50_c10 Dataset & 5000 & 50 & 10 \\
4 & s5000_f50_c50 Dataset & 5000 & 50 & 50 \\
5 & s1000_f500_c10 Dataset & 1000 & 500 & 10 \\
6 & s1000_f500_c50 Dataset & 1000 & 500 & 50 \\
7 & s500_f1000_c10 Dataset & 500 & 1000 & 10 \\
8 & s500_f2000_c50 Dataset & 500 & 2000 & 50 \\
9 & s25000_f20_c50 Dataset & 25,000 & 20 & 50 \\
10 & s50000_f20_c50 Dataset & 50,000 & 20 & 50 \\
\hline
\end{tabular}

seem to be getting formed only after the cluster strictness is set beyond $65 \%$. And with the increase in the strictness value, the number of clusters also seems to be increasing. The same is the case for the other 3 public datasets as well. The additional clusters seem to be getting formed for Wine Dataset, WDBC Dataset, and WPBC Dataset after reaching $60 \%, 40 \%$, and $50 \%$ of cluster strictness, respectively.

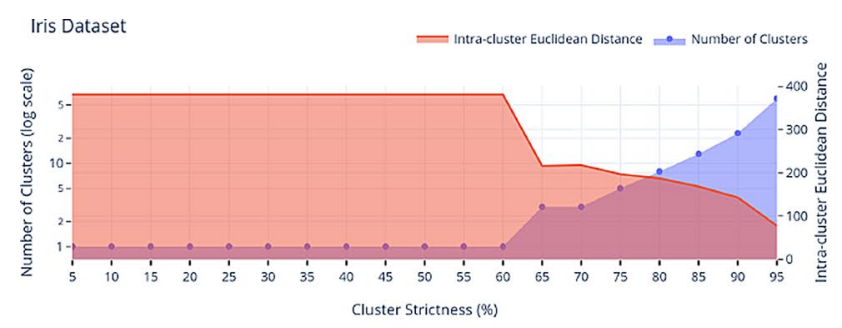

(a) Iris Dataset

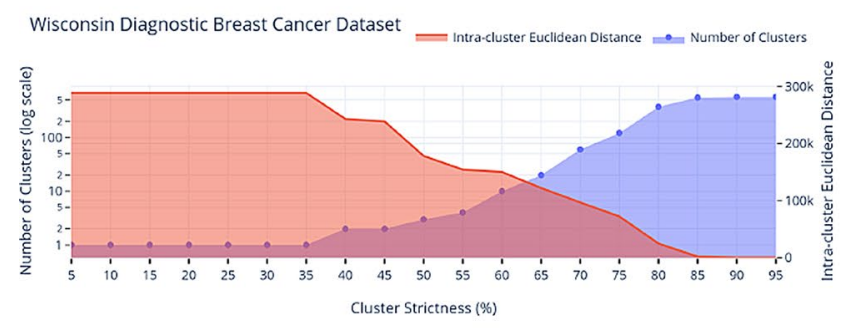

(c) WDBC Dataset
Similarly, the cs-means algorithm was used on 10 isotropic Gaussian blobs. Figure 2 shows the results obtained from those blobs. The s1000_f100_c10 Dataset and s1000_ f100_c50 Dataset had 1000 samples with 100 features, but 10 and 50 centers, respectively. For the former Dataset, the optimal number of clusters showed up when the cluster strictness was in the range $40-65 \%$, and for the latter, the range was $40-65 \%$. Similarly, the number of samples was increased, and the number of features was brought to 50 for generating the s5000_f50_c10 Dataset and s5000_ f50_c50 Dataset, but with 10 and 50 centers, respectively. In the case of these datasets, the former had the optimal number of clusters when the cluster strictness was in the range $40-60 \%$, and for the latter, the range was $45-60 \%$. Similarly, for all the Gaussian blobs, the optimal number of clusters seem to be appearing with cluster strictness in the range $(50 \pm 15) \%$. This condition holds for the public datasets as well. Interestingly, in the case of large samples, s25000_f20_c50 Dataset and s50000_f20_c50 Dataset, the range of cluster strictness for obtaining the optimal number of clusters was too narrow and was seen to be converging at $50 \%$. Summing up, the cluster analysis suggests that the initial lookout value for cluster strictness can be supposed as $50 \%$ while using the cs-means algorithm. Besides, the strictness value can be adjusted accordingly if the task is to group data-points based on allowed proximity of variance within a cluster.

Interestingly two significant deductions can be made from the experimental results. One, the cs-means algorithm seems to be abundantly utilizing the value of cluster strictness in the formation of clusters. Two, the elbow

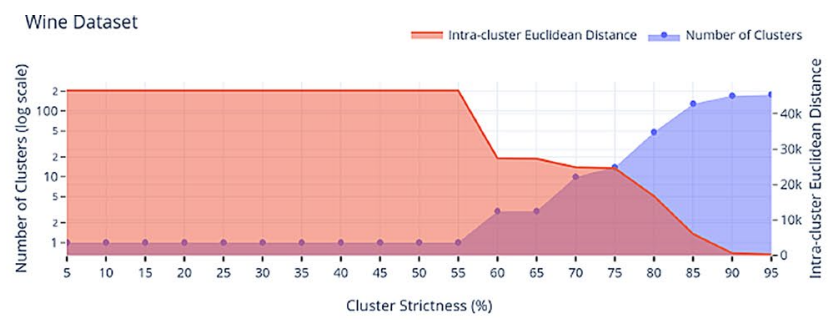

(b) Wine Dataset

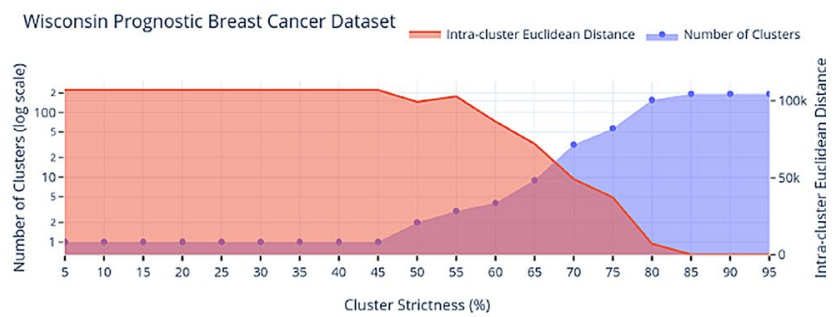

(d) WPBC Dataset

Fig. 1 Results on the 4 Public Datasets 


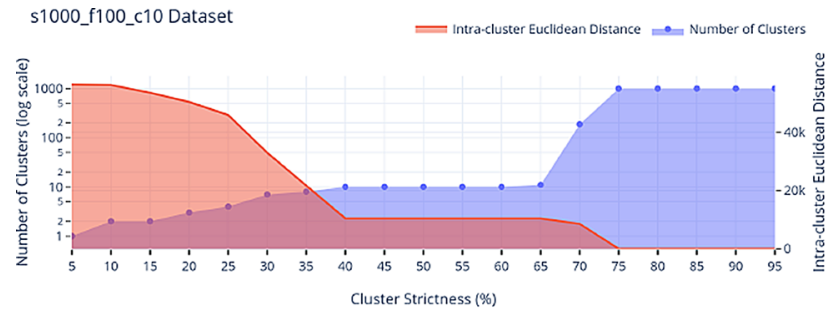

(a) s1000_f100_c10 Dataset

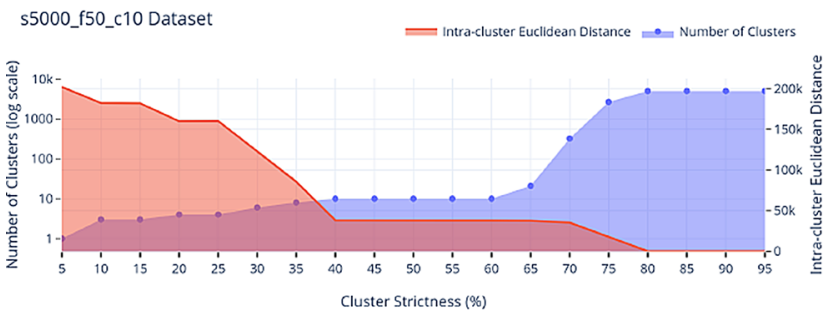

(c) s5000_f50_c10 Dataset

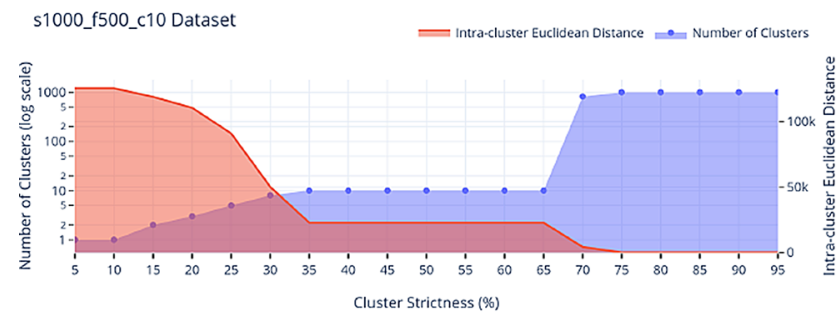

(e) s1000_f500_c10 Dataset

Fig. 2 Results on the 10 Isotropic Gaussian Blobs

of the intra-cluster distance curve can be considered as an ideal location for determining the optimal number of clusters for a dataset. Besides these two deductions, the cs-means algorithm's real-time approach is a significant improvement over traditional clustering algorithms. Algorithms such as K-Means, hierarchical clustering and DBSCAN require all the data-points that are to be grouped, to be already in the vector space. However, the algorithm proposed in this paper takes-in data-points in a serial manner. This real-time behavior contributes to the clustering of the data-points that are yet unseen, but will eventually be available.

\section{Conclusion}

In this paper, a clustering algorithm, cs-means, was proposed, which can cluster data-points without having to specify the number of clusters to be formed. The only hyper-parameter in the algorithm is cluster strictness-the lowest permitted level-of-similarity between a data-point

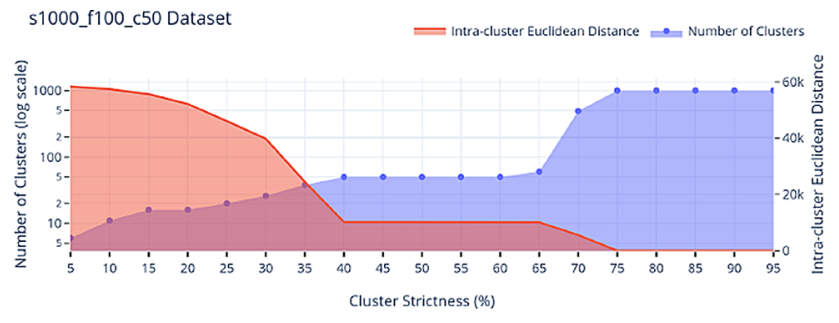

(b) s1000_f100_c50 Dataset

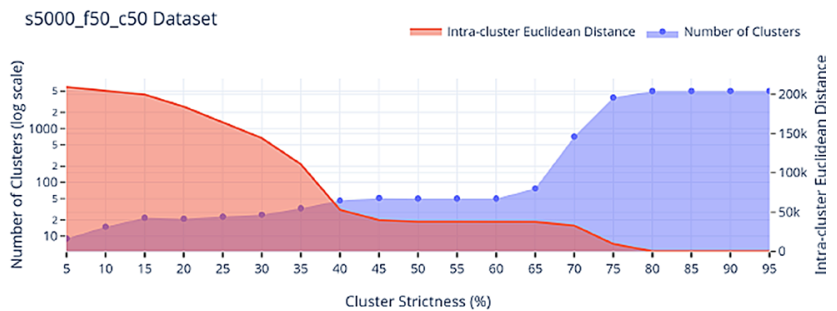

(d) s5000_f50_c50 Dataset

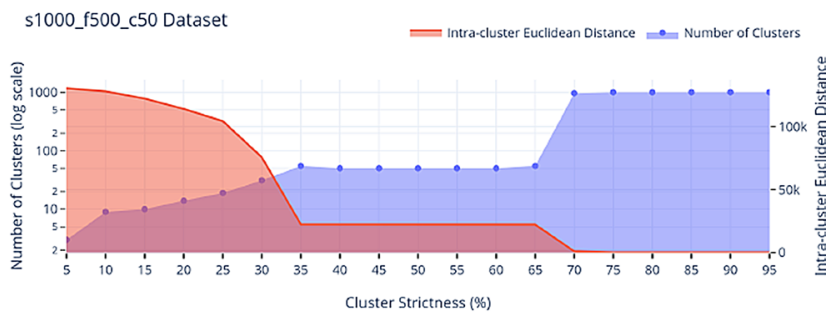

(f) s1000_f500_c50 Dataset

and the centroid of a cluster. The algorithm's primary benefit is that it does not necessarily require all the data-points to be available in the vector space.

In the initial part, the theoretical aspect of the CSmeans algorithm was discussed and implemented on 6 data-points (all of them with 10 dimensions) with the supposition that the data-points are input to the algorithm one after another, just like a stream-of-data in a serial fashion. The experimental results showed that the formation of clusters can be manipulated by increasing or decreasing the value of cluster strictness. It was also evident that cluster-strictness can help identify the optimal number of clusters for a dataset based on the elbow of the intra-cluster Euclidean distance curve. Summing up, the algorithm is applicable in a real-world scenario where the task is to group a stream of data-points, incoming to a system, based on their similarity with the average of features (centroid) of data-points existing in their respective previously formed clusters. If none of the existing clusters satisfy the similarity measure for a new data-point, a new 


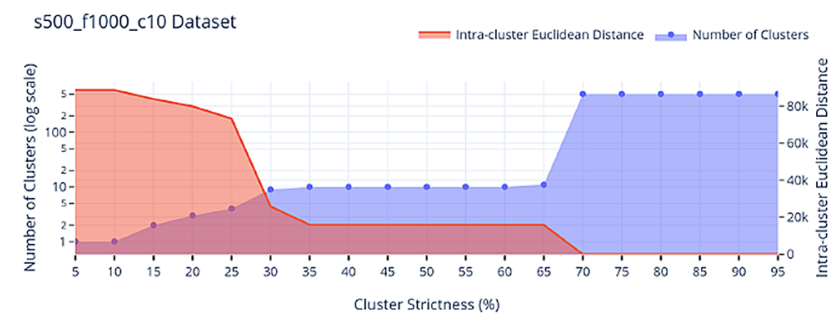

(g) s500_f1000_c10 Dataset

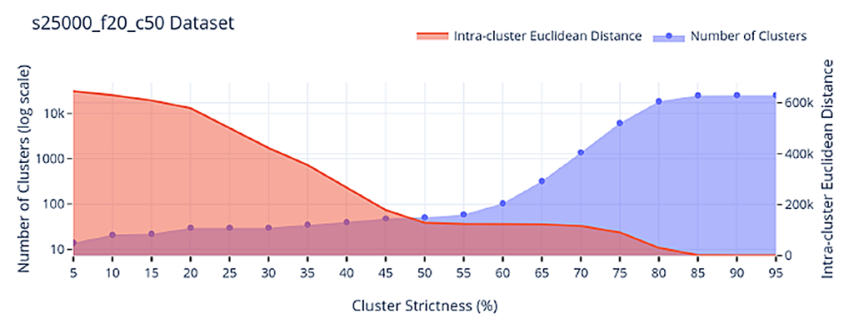

(i) s25000_f20_c50 Dataset

Fig. 2 (continued)

cluster is formed, and the data-point is assigned to the newly formed cluster.

\subsection{Future directions}

When the number of samples and the features are large, the cluster formation becomes time-intensive. Therefore, it can be a new problem, from here, to decrease the number of similarity checks done when new data-points arrive. Instead of checking the similarity of data-points with all the pre-existing clusters, it would reduce the required number of computations if the algorithm can be adjusted to consider only a certain number of clusters for similarity checks.

\section{Compliance with ethical standards}

Conflicts of interest The authors declare that they have no conflict of interest.

\section{References}

1. Aggarwal CC, Philip SY, Han J, Wang J (2003) A framework for clustering evolving data streams. In: Proceedings 2003 VLDB conference. Elsevier, pp 81-92

2. Angelov $P$ (2014) Anomaly detection based on eccentricity analysis. In: 2014 IEEE symposium on evolving and autonomous learning systems (EALS). IEEE, pp 1-8

3. Bezerra CG, Costa BSJ, Guedes LA, Angelov PP (2016) A new evolving clustering algorithm for online data streams. In: 2016

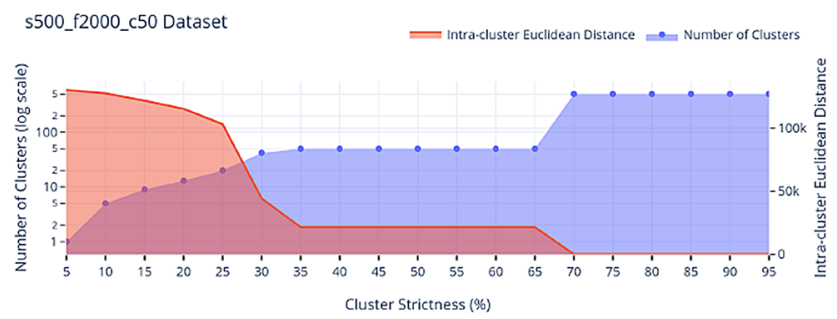

(h) s500_f2000_c50 Dataset

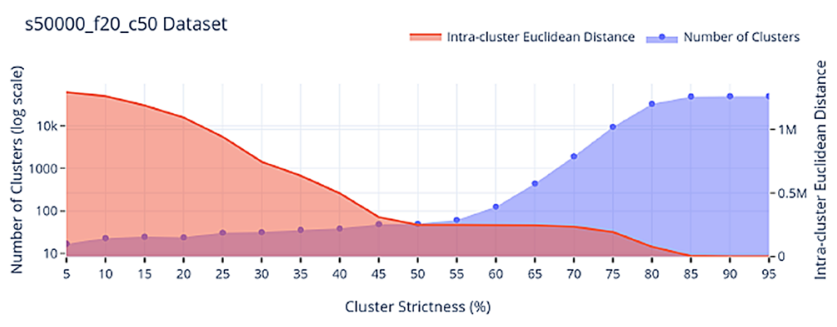

(j) s50000_f20_c50 Dataset

IEEE conference on evolving and adaptive intelligent systems (EAIS). IEEE, pp 162-168

4. Blake CL, Merz CJ (1998) Uci repository of machine learning databases

5. Chen Y, Tu L (2007) Density-based clustering for real-time stream data. In: Proceedings of the 13th ACM SIGKDD international conference on Knowledge discovery and data mining, pp 133-142

6. Das S, Abraham A, Konar A (2007) Automatic clustering using an improved differential evolution algorithm. IEEE Trans Syst Man Cybern Part A Syst Hum 38(1):218-237

7. Ester M, Kriegel HP, Sander J, Xu X et al (1996) A density-based algorithm for discovering clusters in large spatial databases with noise. Kdd 96:226-231

8. Guha S, Mishra N, Motwani R, o'Callaghan L (2000) Clustering data streams. In: Proceedings 41 st annual symposium on foundations of computer science. IEEE, pp 359-366 (2000)

9. Hartigan JA, Wong MA (1979) Algorithm as 136: a k-means clustering algorithm. J R Stat Soc Ser C (applied statistics) 28(1):100-108

10. Hartuv E, Shamir R (2000) A clustering algorithm based on graph connectivity. Inf Process Lett 76(4-6):175-181

11. Kuo R, Suryani E, Yasid A (2013) Automatic clustering combining differential evolution algorithm and k-means algorithm. In: Proceedings of the institute of industrial engineers asian conference. Springer, pp 1207-1215

12. Li X, Liang W, Zhang X, Qing S, Chang PC (2020) A cluster validity evaluation method for dynamically determining the near-optimal number of clusters. Soft Comput 24(12):9227-9241

13. Mur A, Dormido R, Duro N, Dormido-Canto S, Vega J (2016) Determination of the optimal number of clusters using a spectral clustering optimization. Expert Syst Appl 65:304-314

14. Pedregosa F, Varoquaux G, Gramfort A, Michel V, Thirion B, Grisel $\mathrm{O}$, Blondel M, Prettenhofer P, Weiss R, Dubourg V et al (2011) Scikit-learn: machine learning in python. J Mach Learn Res $12: 2825-2830$ 
15. Viloria A, Lezama OBP (2019) Improvements for determining the number of clusters in k-means for innovation databases in SMES. Proc Comput Sci 151:1201-1206

16. Wang M, Abrams ZB, Kornblau SM, Coombes KR (2018) Thresher: determining the number of clusters while removing outliers. BMC Bioinform 19(1):9
17. Xu R, Wunsch D (2005) Survey of clustering algorithms. IEEE Trans Neural Netw 16(3):645-678

Publisher's Note Springer Nature remains neutral with regard to jurisdictional claims in published maps and institutional affiliations. 